\title{
Wilhelmus of Positivity
}

\section{S. S. Kutateladze}

(C) Springer Basel AG 2010

Socially we proceed by example. Wim has exhibited such an example to me, granting a hope of future during the first years of our interest in application of the modern technique of model theory to positivity.

It is worth recalling that in the early 1960s, Wim, then an established analyst of high reputation in Banach lattices, stood the second to Abraham Robinson in promoting the ideas of the brand-new nonstandard analysis. The second publication on nonstandard analysis was Wim's famous article "What is nonstandard analysis?" In 2006 I was asked to give a lecture on the basics of Boolean valued analysis to geometers and of course the title of this lecture was a la Wim "What is Boolean valued analysis?"

It was Wim who edited the famous green book Applications of model theory to algebra, analysis and probability in 1969. A few weeks ago we exchanged letters with Dana Scott who will visit the Malcev Centennial at Novosibirsk later this summer. He reminded me that in this book he published the article "Boolean models and nonstandard analysis" where he forecasted the future of what we know now as Boolean valued analysis.

It so happened that Wim reviewed the majority of books on positivity and nonstandard methods stemming from Novosibirsk and Vladikavkaz, my textbook on functional analysis inclusively. Of course we were and still are very proud of his evaluations and comments. However, I first met Wim in Dresden at Positivity IV and since then a photo of him with Tolya Kusraev and me is always right above my left hand in my study at Novosibirsk.

Wim is a man of an exceptional understanding and marvelous sense of humor. So I finish with a short slightly jocular toast I prepared to welcome Wim here at Positivity VI and sent to him yesterday.

\footnotetext{
S. S. Kutateladze $(\varangle)$

Sobolev Institute, Novosibirsk, Russia

e-mail: sskut@math.nsc.ru
} 
It is an honor and pleasure for me to felicitate Professor Luxemburg who is unfortunately absent from this Positivity meeting.

We are just a bunch of positivity adepts. Thinking positively, we may view the realm of mathematics as the free quite real vector space over all mathematicians. The conic hull of all positivity humans makes mathematics into an ordered vector space. Most likely this yields a vector lattice despite the ludicrous claims of some fellow mathematicians to be the top elements in this ordered vector space. In any case, we belong to the positive cone of mathematics and so we are positive. Since positivity is a general mode of living, it is better to say that a member of the positive cone has positive sense. As usual in many dimensions, most vectors are senseless, i.e., the relevant persons have no sense at all.

Professor Luxemburg has taught us that we live not only in the vector lattice of mathematics but also in the unusually nonstandard universe where we must distinguish between the negligible and the eternal as well as between the infinite and the infinitesimal.

Positivity is a relation and some of us are more positive than the others. In the nonstandard world we live in, one is definitely greater than the other, if the difference between the two is infinitely large.

Professor Luxemburg occupies an exceptional place in positivity, for he is definitely greater than any standard mathematician of positive sense.

Professor Luxemburg's service to mathematics is so impressive that I am sure that no one but he has any right to repeat the ancient words of Het Wilhelmus: "Life and my all for others I sacrificed, for you!"

The noble tradition calls us to raise our glasses and wish Professor Luxemburg, the true Wilhelmus of Positivity, many happy returns of the day.

Inspiration is always genuine. You cannot counterfeit inspiration. We all share the feeling of inspiration that stems from Wim. 